
治疗中的应用

\author{
何文亚, 邹艳*, 郑蒙, 师冰洋* \\ 河南大学生命科学学院, 河南大学-麦考瑞生物医学联合创新中心, 开封 475004 \\ *通讯作者, E-mail: yzou@henu.edu.cn; bs@henu.edu.cn
}

收稿日期: 2019-06-03; 接受日期: 2019-07-04; 网络版发表日期: 2019-07-19

国家重点研发计划(编号: 2018YFA0209800)和国家自然科学基金(编号: NSFC 31600809, 31800841, U1604177, U1804139)资助

\begin{abstract}
摘要纳米药物在癌症的精准医疗方面具有广阔的应用前景，但纳米材料易被机体免疫系统识别并清除的特 性使得其在癌症治疗方面的应用存在很大的局限性.受自然界生物系统的启发, 生物细胞介导的药物递送系统近 年来得到了广泛关注. 该技术通过将生物体内源性细胞膜作为功能材料包覆在纳米药物表面, 赋予其细胞膜的天 然属性，或者将纳米载药粒子直接与活细胞共粰育，制备载药细胞，有效地将生物体“自体”的性质与“人工”纳米 材料的优势相结合. 这不仅大大降低了纳米药物的免疫原性, 延长其血液循环时间, 而且还可使其具备更强的肿 瘤靶向能力. 本文初步探讨了生物细胞仿生药物递送系统在肿瘤治疗中的研究进展并对其未来研究进行展望.
\end{abstract}

关键词仿生修饰, 纳米药物, 细胞, 癌症治疗

\section{1 引言}

癌症已经成为威胁人类健康的头号杀手. 目前, 临 床上癌症的治疗以手术切除为基础，但由于肿瘤的易 转移性及特定肿瘤发病部位的敏感性，单一的手术切 除通常不能达到理想的治疗效果，因此化疗或术后辅 以化疗成为癌症治疗必不可少的手段之一. 化疗即直 接对人体注射抗癌药物, 是一种全身性的治疗手段, 由于其对细胞不具有选择性, 在杀死癌细胞的同时也 会杀死大量正常细胞，因此会对机体造成很大的副作 用 ${ }^{[1]}$. 此外, 化疗药物在血液中的半衰期时间短, 加之 受到肿瘤基质的阻碍和肿瘤内部高压环境的影响，化
疗药物很难渗透进入实体瘤内部，当肿瘤部位药物的 浓度低于某一临界值时，药物将起不到有效杀死肿瘤 细胞的效果. 第三，长期化疗易使癌细胞产生耐药性, 并且多数化疗药物难以跨越特定的生理屏障(如血脑 屏障), 因而无法实现对颅内肿瘤的有效治疗 ${ }^{[2,3]}$. 因此, 开发安全高效的药物载体以提高化疗效率对肿瘤的治 疗具有重要的意义.

基于纳米技术的载药系统的出现为抗癌药物的靶 向递送提供了新思路. 纳米载药体系具有高的载药效 率和良好的生理稳定性, 可通过被动或主动靶向作用 在肿瘤部位富集, 并在肿瘤微环境(低 $\mathrm{pH}$, 还原性)或 外部条件(光照、磁场)的刺激下使药物缓慢持续释放,

引用格式: He W, Zou Y, Zheng M, Shi B. Cell-derived biomimetic drug delivery systems for cancer therapy. Sci Sin Chim, 2019, 49: 1203-1212, doi: 10.1360/ SSC-2019-0068 
不仅提高了药物的生物利用度，而且可以降低其对正 常组织的损伤. 纳米药物的独特性质使其在癌症治疗 领域显示出广阔的应用前景 ${ }^{[4-6]}$. 但是作为一种外来物 质，纳米材料在生物体内不可避免地会引起免疫系统 的排斥反应. 此外, 当纳米药物进入血液循环之后, 机 体的网状内皮系统会识别这些外来物质并将之快速清 除出体外，降低其血液循环时间. 为解决上述问题，当 前最常用的方法是将纳米粒子表面进行聚乙二醇(即 PEG)修饰。该方法一方面能增加纳米药物的稳定性; 另一方面也能够使其具有“隐蔽性”，从而避免被网状 内皮系统识别. 但是, 有研究发现, 机体会对PEG化纳 米载体产生抗PEG免疫反应，使得二次注射的纳米粒 子被更快地清除 ${ }^{[7 ~ 10]}$. 受自然界生物系统的启发, 生物 细胞介导的药物递送系统近年来得到了广泛的关注. 该策略通过将内源性细胞膜(如红细胞膜、血小板、 免疫细胞膜、癌细胞膜及杂化细胞膜)作为功能材料 包覆在纳米药物表面, 赋予其细胞膜的天然属性, 或者 将纳米载药粒子直接与活细胞共孵育, 制备载药细胞, 可有效地将生物体“自体”的性质与“人工”纳米材料的 优势有机结合(图1). 这一方面可以改善纳米药物在活 体内的药代动力学性质，使其具有体内长循环、免疫 逃逸等特性，另一方面也赋予纳米药物更强的肿瘤靶 向能力，使其在肿瘤的精准医疗领域具有广阔的应用 前景 ${ }^{[1117]}$. 本文以细胞为标准, 分类介绍基于生物体 细胞的仿生载药体系的设计构建及其在肿瘤的化学治 疗、免疫治疗、光热/光动治疗及多模式协同治疗中 的应用.

\section{2 红细胞仿生药物递送体系}

基于红细胞的仿生修饰是当前研究最为成熟的纳 米载药体系表面改性方式之一. 红细胞是血液中含量 最丰富的细胞，红细胞膜表面含有多种蛋白(如膜蛋 白、CD47蛋白)及多糖，这些成分可以抑制免疫系统 对红细胞的识别摄取, 使红细胞在体内维持长时间的 循环(其在血液中的循环寿命可达四个月). 因此, 用红 细胞膜包覆纳米粒子可大大增加其免疫相容性. 此外, 细胞膜磷脂层的流动性也使得对红细胞膜表面进行肿 瘤靶向性及多功能性修饰成为可能 ${ }^{[18 \sim 21]}$.

Liu课题组 ${ }^{[22]}$ 报道了利用红细胞膜包裹负载抗癌 药物阿霉素的介孔普鲁士蓝纳米粒子，构建仿生纳米

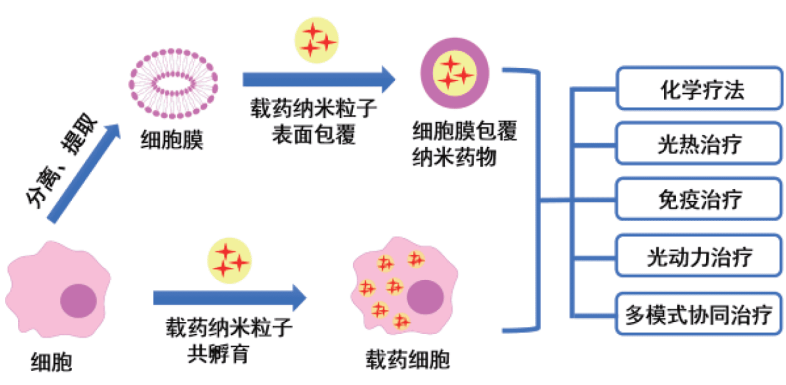

图 1 细胞仿生药物递送系统的制备过程示意图及其在癌 症治疗领域的应用(网络版彩图)

Figure 1 Schematic illustration of the fabrication of the cell-derived biomimetic drug delivery systems and their application in cancer therapy (color online).

药物用于肿瘤的治疗. 实验结果表明，相对于没有经 过修饰的纳米粒子，红细胞膜包覆的纳米载药粒子能 够有效地避免巨噬细胞的吞噬作用，从而明显延长其 在血液中的循环时间. 此外, 该纳米载体还具有高的 载药效率(载药量可达 $130 \%$ (质量百分比))和光热治疗 能力, 当到达肿瘤部位时, 能够在肿瘤处微酸性条件和 近红外光照射下实现药物的有效释放，达到良好的协 同抗肿瘤效果.

尽管红细胞膜的修饰能够延长纳米载药体系的血 液循环时间, 但是红细胞自身并不具有肿瘤靶向性. 大 量的研究结果表明，在纳米粒子表面修饰具有肿瘤靶 向功能的基团, 能够显著提高纳米材料的肿瘤靶向能 力. Sun等 ${ }^{[23]}$ 提出了在红细胞膜的磷脂双分子层中插 入肿瘤靶向基团RGD, 构建出具有肿瘤主动靶向性的 红细胞. 体外及活体内实验结果表明, 靶向基团的引 入没有对红细胞的生理机能造成明显的影响. 之后他 们又通过低渗透析法在靶向修饰的红细胞内部包载阿 霉素和光热试剂ICG, 所得载药红细胞不仅表现出了 更强的与癌细胞结合的能力, 并且在近红外光照射下, ICG吸收光能产生热量促使红细胞破裂释放出阿霉素, 对癌细胞表现出了强的杀伤作用.

相对于其他部位的肿瘤, 如脑肿瘤, 因其发病部位 特殊, 因此治疗难度更高. 用于脑肿瘤的化疗药物通常 需要具备两个基本条件: (1) 药物能够穿越血脑屏障到 达肿瘤部位; (2) 药物可以有效地被肿瘤细胞摄取. 当 前临床上常用的化疗药物(如阿霉素、顺铂)及大多数 已报道的纳米载药体系不具备穿越血脑屏障的能力. 为了解决这一难题, 本课题组提出了在红细胞膜表面 修饰能够特异性靶向低密度脂蛋白受体的Angiopep-2 
配体, 并用于包覆负载了阿霉素和Lexiscan (瞬时打开 血脑屏障)的 $\mathrm{pH}$ 敏感性聚合物纳米粒子, 构建智能型纳 米载药粒子(Ang-RBCm@NM-(Dox/Lex))用于原位脑 胶质瘤的治疗 ${ }^{[24]}$ 。低密度脂蛋白受体在血脑屏障及胶 质瘤细胞(U87MG)表面均过表达，因此，所得纳米载 药体系可通过受体介导的胞吞作用穿过血脑屏障将药 物递送至肿瘤部位. 此时, 肿瘤部位的微酸性环境能够 触发载药纳米粒子释放药物, Lexiscan的溶出促使血 脑屏障打开，进一步增强载药纳米粒子跨越血脑屏障 的能力. 在肿瘤部位, 癌细胞表面的Angiopep-2受体与 纳米药物表面的配体结合, 可有效地摄取纳米药物. 在 癌细胞内部, 溶酶体内的酸性环境引发纳米粒子解体, 释放所负载的抗癌药物, 从而可将癌细胞杀死. 小鼠荷 瘤动物模型实验结果表明，该纳米载药粒子具有长的 血液循环时间，能够跨越血脑屏障在胶质瘤部位靶向 富集，起到了良好的抑制肿瘤生长的效果，显著延长 了荷瘤小鼠的生存周期(图2)。此外, 该纳米载药粒子 还具有良好的生物相容性和安全性，通过尾静脉注射 进入小鼠体内之后，没有对小鼠的脏器及各项生理体 征造成明显的影响, 显示出其潜在的临床应用价值.
免疫治疗是继常规的手术、放疗和化疗之后的一 种新兴的癌症治疗方式, 它主要借助于增强患者自身 免疫系统的功能来识别和杀死肿瘤细胞，是当前肿瘤 治疗领域的研究热点 ${ }^{[25,26]}$. Guo等 ${ }^{[27]}$ 报道了将红细胞 膜作为肿瘤相关抗原肽递送载体构建纳米疫苗用于黑 色素瘤的免疫治疗. 该纳米疫苗由甘露糖功能化的红 细胞膜包覆连接有黑色素瘤抗原多肽的还原响应性聚 乳酸-羟基乙酸共聚物(PLGA)纳米粒子构成. 甘露糖 基团的修饰可以增强淋巴部位抗原提呈细胞对该纳米 疫苗的摄取能力, 在抗原提呈细胞(树突状细胞)内部, 纳米粒子在由谷胱甘肽等还原性物质的作用下解离并 释放出抗原多肽, 促进树突状细胞的成熟, 进而使机体 产生免疫反应以抑制肿瘤的生长. 动物模型实验结果 表明，该纳米疫苗不仅能使小鼠产生抗肿瘤免疫，预 防肿瘤的发生, 并且能够抑制黑色素瘤的生长和转移. 该工作为抗肿瘤疫苗的研发提供了新的思路.

此外, $\mathrm{GaO}$ 等 ${ }^{[28]}$ 还报道了利用红细胞膜包覆负载 有具有携氧能力的全氟碳的PLGA纳米粒子，制备出 粒径约 $290 \mathrm{~nm}$ 的人工红细胞，该纳米药物可通过被动 靶向作用到达肿瘤部位，改善肿瘤部位的缺氧状况，

(a)
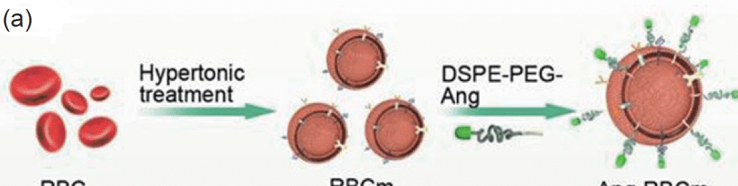

RBC

$\mathrm{RBCm}$

Ang-RBCm

pH-sensitive

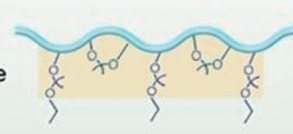

a-dextran
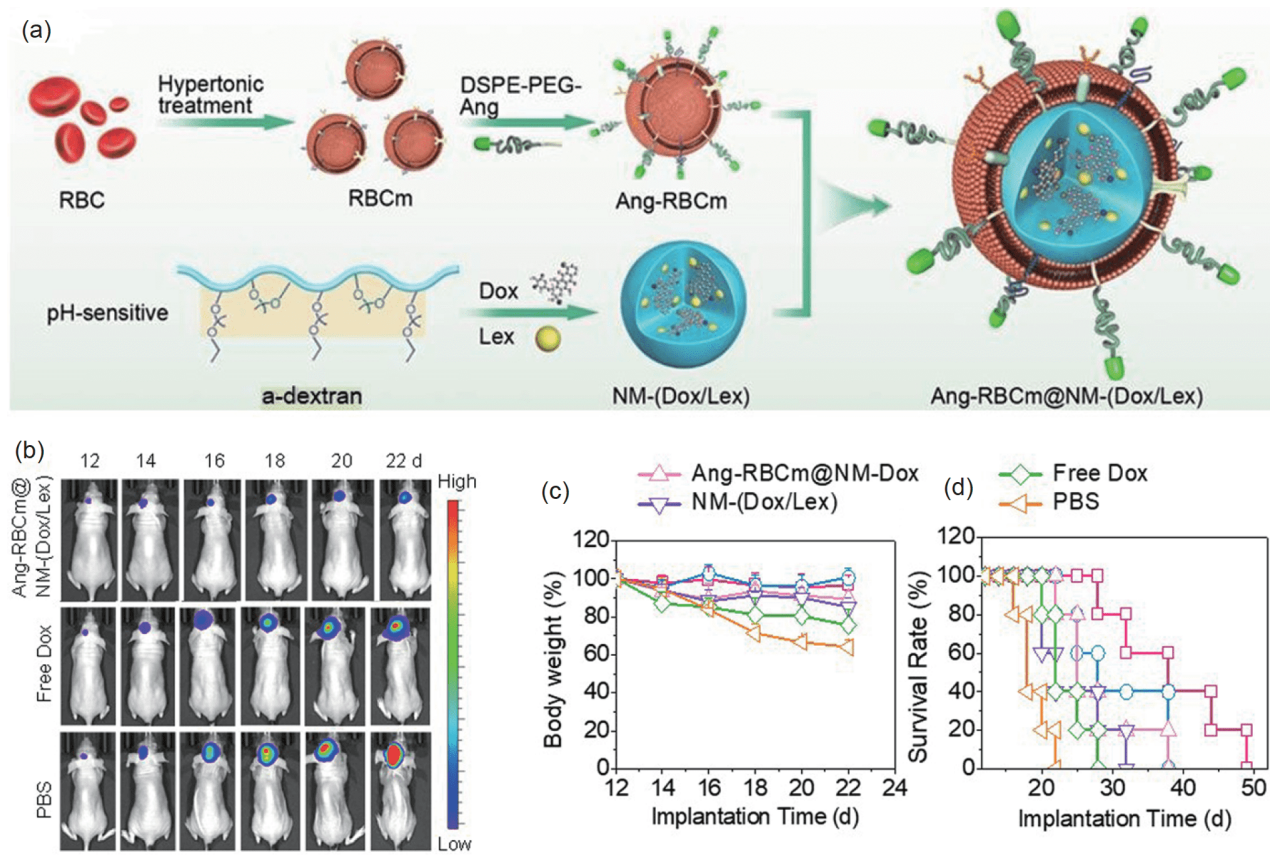

图 2 (a) Ang-RBCm@NM-(Dox/Lex)的制备过程示意图; (b) 接受PBS、阿霉素(DOX)及Ang-RBCm@NM-(Dox/Lex)注射的 原位脑胶质瘤小鼠的肿瘤苂光成像图; 不同实验组荷瘤小鼠的体重变化情况(c)及生存周期考察 $(\mathrm{d})^{[24]}$ (网络版彩图)

Figure 2 (a) Schematic illustration of the fabrication of Ang-RBCm@NM-(Dox/Lex); (b) in vivo fluorescence images of orthotopic human glioblastoma tumor bearing mice following treatment with PBS, DOX, and Ang-RBCm@NM-(Dox/Lex) at different time intervals. The evaluation of the body weight variation (c) and survival rate (d) of tumor-bearing mice in different experimental group [24] (color online). 
提高肿瘤对放疗的敏感性.

\section{3 血小板仿生药物递送体系}

血小板是血液中的循环哨兵，能够靶向富集于血 管损伤部位, 阻碍血栓形成, 维持正常的血液循环. 此 外，血小板与肿瘤的发生发展也有着密不可分的关系. 研究表明, 肿瘤细胞在生长和转移的过程中会活化和 诱导血小板在其周围聚集，以帮助其逃避机体免疫系 统的攻击，同时保护肿瘤细胞免受血流等外力的损 害 ${ }^{[29-31]}$ (图3). 血小板与肿瘤细胞相互识别和相互作用 的特性，使得构建基于血小板的抗肿瘤“特洛伊木马” 用以靶向递送抗癌药物成为可能.

Gu课题组 ${ }^{[32]}$ 报道了将血小板细胞膜表面进行肿 瘤坏死因子相关凋亡诱导配体(TRAIL)修饰，用于制 备仿生纳米药物的工作. 该纳米药物内核由负载了阿 霉素的酸敏感纳米凝胶构成，借助于血小板表面的 $\mathrm{p}$-选择素能够特异性与肿瘤细胞表面过表达的 CD47 受体结合的特性，该纳米粒子能够有效地靶向病灶处 和血液中的(循环肿瘤细胞)乳腺癌细胞. 在癌细胞表 面，纳米粒子表面的TRAIL配体通过结合细胞表面的 死亡受体DR4、DR5诱导细胞发生调亡; 当其进入癌 细胞之后，在细胞内部的酸性环境下，纳米凝胶粒子 解体释放出阿霉素，进一步诱发细胞凋亡．体内外实 验结果表明，该载药纳米粒子对肿瘤的生长和转移均 能起到良好的抑制作用.
术后复发是传统癌症治疗手段的弊端之一，术后 巩固治疗对达到理想的癌症治疗效果具有重要的意 义. Zhang等 ${ }^{[33]}$ 利用血小板能在伤口富集止血的属性, 构建出表面表达PD-1受体的血小板用于清除术后残 存的癌细胞. 癌细胞表面的PD-L1配体与免疫细胞 $\left(\mathrm{CD} 8^{+} \mathrm{T}\right.$ 细胞) 表面的PD-1受体结合并使 $\mathrm{CD} 8^{+} \mathrm{T}$ 细胞快 速耗竭, 是导致免疫治疗失败的重要原因. 在该工作 中，他们通过基因工程的方式在体外改造MK巨噬细 胞，并诱导其产生表面表达PD-1受体的血小板. 所得 血小板由尾静脉注射方式进入小鼠体内之后，能够靶 向手术切除后伤口处残余的癌细胞并与其表面的 PD-L1配体结合, 阻断PD-1/PD-L1通路, 逆转 $\mathrm{CD} 8^{+}{ }^{+}$细 胞耗竭, 从而抑制肿瘤的复发. 此外, 该血小板还能负 载抗癌药物环磷酰胺, 有效地杀灭肿瘤周围的调节性 $\mathrm{T}$ 细胞(Treg细胞), 进一步恢复 $\mathrm{CD} 8^{+} \mathrm{T}$ 细胞的活性.

\section{4 免疫细胞仿生药物递送体系}

巨噬细胞和中性粒细胞是机体内重要的免疫细胞, 参与机体的炎症反应. 研究证实, 肿瘤的发生和转移与 不可控制的炎症有密切的关系, 肿瘤细胞在生长过程中 会分泌一系列炎症因子和趋化因子，如TNF- $\alpha$ 、IFN- $\gamma$ 及IL-6, 招募免疫细胞在其周围组织富集. 此外, 作为肿 瘤微环境的重要构成部分, 免疫细胞在肿瘤的转移发展 中也起着举足轻重的作用 ${ }^{[34-37]}$ (图3). 因此, 免疫细胞也 被广泛用来构建抗肿瘤仿生纳米药物.
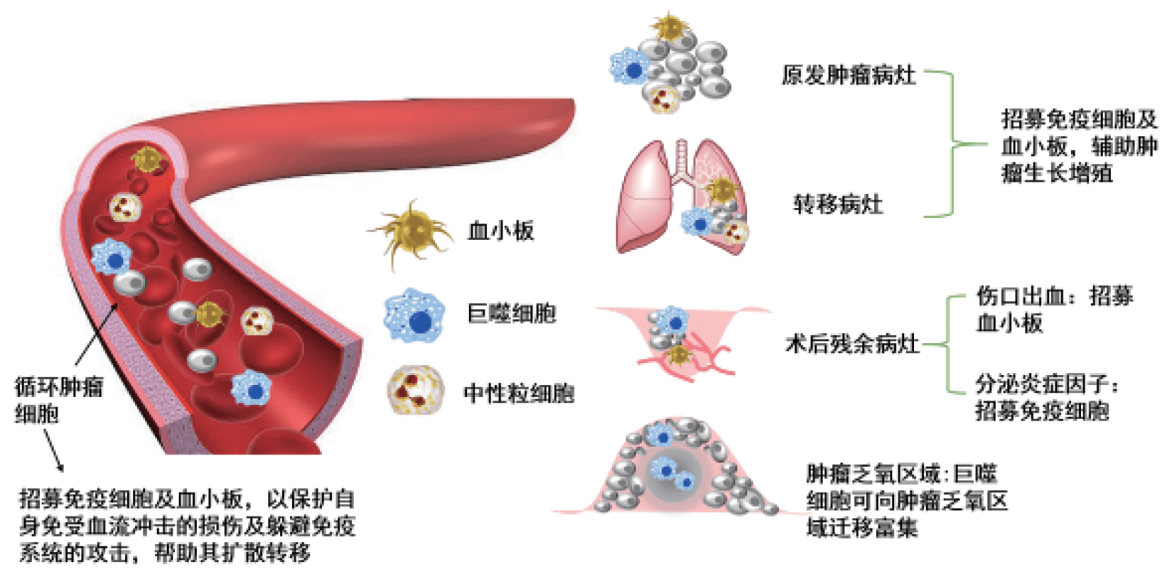

肿瘦乏氧区域:巨喘

细胞可向肿溜乏氧区

域迁移富葉

图 3 血小板及免疫细胞与肿瘤生长增殖有密切的关系. 基于此，可构建基于血小板及免疫细胞的仿生纳米药物用于肿瘤的 靶向、精准治疗(网络版彩图)

Figure 3 Platelet and immune cells are closely related to tumor's proliferation and metastasis, based on which platelet- and immune cell-derived biomimetic nano-drugs can be fabricated for targeted therapy of tumor (color online). 
当肿瘤发生时，机体会做出募集大量外周血单核 细胞至肿瘤部位的反应, 随后单核细胞会逐渐分化变 成巨噬细胞 (又称为肿瘤相关巨噬细胞), 并逐渐向肿 瘤乏氧部位迁移. 利用该特点, Yang课题组 ${ }^{[38]}$ 将巨噬 细胞RAW264.7与金纳米棒共孵育, 得到装载有金纳 米棒的RAW264.7细胞. 实验结果表明, 金纳米棒的装 载不会对RAW264.7细胞的活性和生理功能造成影响. 由尾静脉注射进入小鼠体内之后, 借助于光声成像手 段可观察到RAW264.7细胞能够有效地在肿瘤乏氧部 位富集, 并在近红外光照射下杀死肿瘤细胞, 抑制肿瘤 的生长. 在肿瘤部位, 单核细胞分化所形成的巨噬细胞 会过表达豆荚蛋白，而该蛋白酶在单核细胞中不表达. $\mathrm{He}$ 等 ${ }^{[39]}$ 用单核细胞装载豆荚蛋白敏感性的聚合物载 药纳米粒子, 该载药细胞能够主动靶向乳腺癌转移病 灶，随后单核细胞分化并释放出所载抗癌药Mertansine. 动物实验结果表明, 该纳米药物对乳腺癌转移抑 制率可达 $77.8 \%$. 此外, 该载药体系精准的药物释放机 制也有效避免了其在体内药物泄露的问题.

中性粒细胞是哺乳动物中最重要的一种白细胞, 它能准确地迁移至急性损伤组织和炎症部位, 在机体非 特异性免疫中起重要的作用 ${ }^{[40,41]}$. 肿瘤组织在本质上也 是一种炎症, 被炎症反应激活的中性粒细胞膜表面的黏 附分子使得其对肿瘤病灶和血液中的循环肿瘤细胞都 具有较好的靶向作用 ${ }^{[42,43]}$. Kang 等 ${ }^{[44]}$ 从血液中分离出 中性粒细胞，并在体外诱导其产生炎症应激反应，然后 通过离心的方法得到表面过表达黏附分子的中性粒细 胞膜并将其用于包覆负载了抗肿瘤抑制剂卡菲佐米的 PLGA纳米粒子. 所得纳米载药粒子不仅对小鼠血液中 的循环乳腺癌细胞表现出强的杀伤作用, 并且能够有效 地抑制淋巴部位及肺部早期乳腺癌病灶的产生.

除了具有良好的炎症靶向性，中性粒细胞还能够 穿过血脑屏障靶向脑部肿瘤. Xue 等 ${ }^{\left[{ }^{[5]}\right]}$ 将分离纯化得 到的中性粒细胞与负载了抗癌药物紫杉醇的脂质体纳 米粒子共睬育, 构建出载药中性粒细胞(PTX-CL/NEs) 用于脑肿瘤的术后辅助治疗. 接受手术切除之后, 小鼠 的伤口部位会产生大量的炎症因子，这些炎症因子能 够诱导载药中性粒细胞穿过血脑屏障到达脑肿瘤部 位. 随后, 肿瘤部位丰富的炎症因子将导致载药中性粒 细胞被过度活化并释放出中性粒细胞胞外诱捕网，同 时载药脂质体纳米粒子被释放到周围环境中, 随即可 被癌细胞摄取并杀死癌细胞, 起到了良好的抑制脑肿
瘤复发的效果.

\section{5 癌细胞仿生药物递送体系}

相对于正常细胞, 癌细胞有着独特的特点, 如无限 增殖、抗调亡及免疫逃逸, 这些特性都与癌细胞表面 的蛋白和受体密不可分. 此外, 癌细胞表面的抗原还 具有同源或异源黏附特性, 在肿瘤的生长和转移过程 中发挥着聚集细胞的作用 ${ }^{[46,47]}$. 近年来, 通过利用癌 细胞的同源靶向性和免疫逃逸功能构建靶向性仿生纳 米载药体系的研究也吸引了人们越来越多的关注.

Li课题组 ${ }^{[48]}$ 报道了用小鼠乳腺癌细胞膜包覆负载 了紫杉醇的聚己内酯纳米粒子, 细胞膜的包覆一方面 提高了载药纳米粒子的药物缓释性能, 另一方面也赋 予其体内长循环和乳腺癌肿瘤靶向的特性. 经尾静脉 注射进入小鼠体内之后, 该仿生纳米载体对小鼠乳腺 癌的原发病灶和血管转移病灶均具有较好的靶向效 果, 起到了显著的抑制肿瘤生长的效果.

Yang 等 ${ }^{[49]}$ 通过液氮冷冻法提取小鼠黑色素瘤细胞 膜, 所得细胞膜完整地保留了其表面的膜蛋白和各种肿 瘤相关抗原分子, 随后通过脂质插入法在膜表面修饰甘 露糖基团, 用以包覆负载了小分子免疫调节剂的PLGA 纳米粒子构建抗肿瘤疫苗. 该纳米疫苗由皮内注射后能 够逐渐迁移至小鼠淋巴部位, 靶向树突状免疫细胞并激 发其产生抗肿瘤免疫反应, 抑制肿瘤的产生. 此外, 通过 与肿瘤免疫检查点抑制剂联合使用, 该纳米疫苗对已经 产生的肿瘤也具有较好的治疗效果.

Zhang课题组 ${ }^{[50]}$ 采用癌细胞膜修饰负载了葡萄糖 氧化酶和过氧化氢酶的锌-卟啉金属有机框架材料, 构 建出纳米生物级联反应器( $\mathrm{mCGP}$ ) 用于肿瘤的饥饿和 光动力治疗 (图4). 所得纳米药物被癌细胞摄取之后, 在过氧化氢酶的催化作用下, 癌细胞内高含量的过氧 化氢分解产生氧气, 氧气的产生一方面会促进葡萄糖 氧化酶对葡萄糖的氧化分解, 消耗癌细胞内的葡萄糖 以降低其生长所需的能量供应, 另一方面也会增强卟 啉在光照条件下产生单线态氧自由基的能力, 在小鼠 动物模型实验中起到了良好的抑制肿瘤生长的效果.

\section{6 其他类型细胞仿生药物递送体系}

干细胞是一种具有自我复制能力的多潜能细胞, 

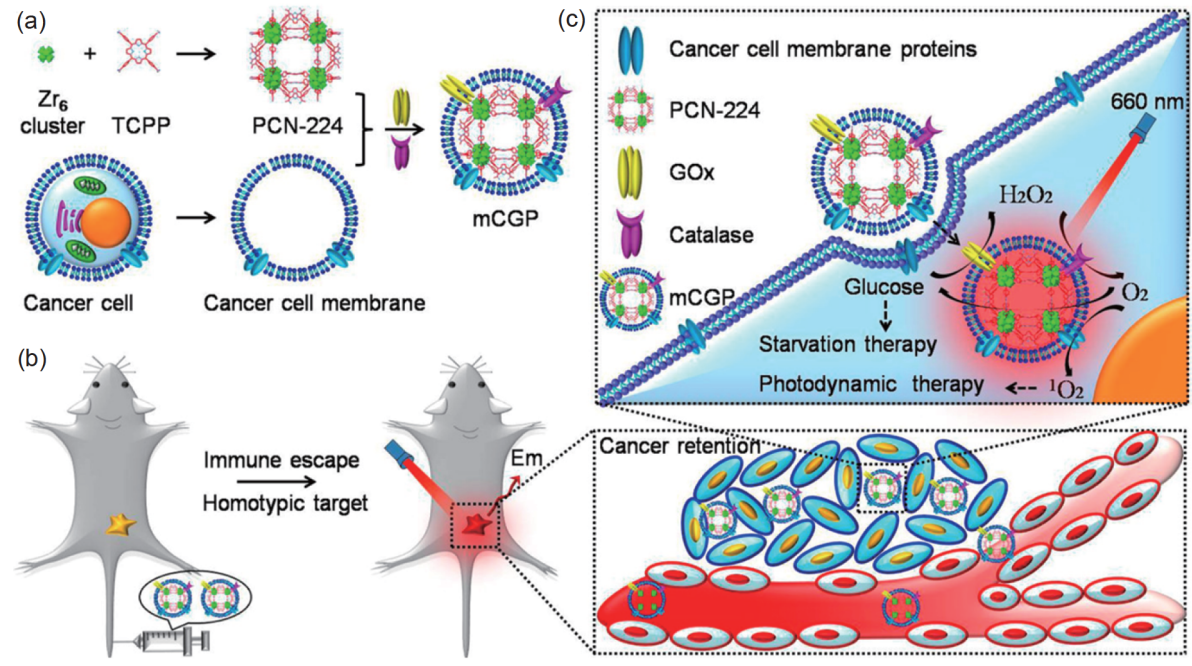

图 4 (a) mCGP的合成示意图; (b) mCGP的免疫逃逸性能和同源靶向性使得其能够通过血液循环在肿瘤部位富集; (c) 在癌细 胞内部mCGP消耗葡萄糖, 并在光照条件下产生单线态氧的过程示意图 ${ }^{[50]}$ (网络版彩图)

Figure 4 (a) Schematic illustration of mCGP; (b) the immune escape and homotypic targeting abilities of mCGP allowing it to accumulate in tumor site following intravenously injection; (c) schematic that shows mCGP cuts off the glucose supply and promotes ${ }^{1} \mathrm{O}_{2}$ generation under light irradiation in cancer cells [50] (color online).

并且具有低的免疫原性. 研究表明, 肿瘤细胞分泌的细 胞因子和趋化因子可以诱导干细胞向肿瘤组织富 集 ${ }^{[51,52]}$. 因此许多类型的干细胞, 如骨髓间充质干细胞 也被用来构建肿瘤靶向性纳米药物. Jiang课题组 ${ }^{[53]}$ 通 过低渗处理的方式提取间充质干细胞膜, 并用其包覆 携带siRNA的氧化铁纳米粒子，用于肿瘤的光热/基因 协同治疗. 实验结果表明, 所得纳米药物能够靶向递 送siRNA至小鼠皮下移植瘤部位，并引发癌细胞发生 调亡，结合纳米氧化铁的光热治疗效果，该纳米药物 起到了较好的抗肿瘤效果. Menei课题组 ${ }^{[54]}$ 将人源间 充质干细胞分别与载药聚合物纳米粒子和载药脂质体 共孵育, 发现两种载药纳米粒子都能被干细胞摄取, 并 且证实纳米粒子的装载不会对干细胞的活力和分化能 力造成影响. 所得载药干细胞经由瘤内注射进入脑胶 质瘤小鼠体内之后，能够靶向迁移至肿瘤与正常脑组 织的边缘处, 而不会进入周围正常的脑组织, 该工作 证实干细胞可以作为一种有效的药物载体递送药物至 脑胶质瘤. 近年来越来越多的研究证实干细胞具有穿 越血脑屏障的能力 ${ }^{[55,56]}$, 基于干细胞的纳米载药体系 必将对包括肿瘤在内的脑部疾病的治疗带来转机.

细菌是一种天然的免疫佐剂, 可诱导机体产生炎 性因子来增强机体的免疫反应. 此外, 它还是一种良 好的基因转染载体，19世纪末期开始，研究者们逐渐
发现细菌(如沙门氏菌)也具有肿瘤靶向和抗肿瘤的功 能. 目前, 利用减毒沙门菌作为抗肿瘤核酸药物( $\mathrm{siR}$ NA、质粒等)运载体系已经在动物实验及临床I、II期 试验中取得了较好的效果 ${ }^{[57 ~ 60]}$. 2016年，Martel课题 组 ${ }^{[61]}$ 创新性地提出了用磁性-厌氧细菌MC-1 作为药物 载体用于抗癌药物的靶向递送, 他们通过共价键合的 方式在细菌表面负载载药纳米脂质体, 所得载药细菌 可以在外加磁场的作用下迁移至小鼠肿瘤部位, 并且 由于MC-1细菌厌氧的特性, 当撤去外加磁场之后载药 细菌可以向肿瘤内部的乏氧区域扩散. 实验结果表明, 该细菌药物递送系统具有高的递送效率, 在磁场的作 用下, 约 $55 \%$ (总注射量: $1 \times 10^{8}$ 个, 肿瘤部位局部注射) 的细菌可以有效地靶向至肿瘤乏氧部位(图5).

外泌体是由细胞分泌的粒径为 $50 \sim 150 \mathrm{~nm}$ 的小膜 泡, 又称为“天然的纳米粒子”. 和细胞一样, 外泌体具 有低的免疫原性、巨噬细胞吞噬逃逸特性及良好的生 物相容性 ${ }^{[62,63]}$. 此外, 外泌体还具备穿越特定生物屏障 如细胞质膜及血脑屏障等特性 ${ }^{[64]}$. 近年来, 外泌体也 被广泛地应用于化疗药物、特定功能的核酸及蛋白质 的递送载体用于肿瘤的治疗 ${ }^{[65 ~ 69]}$.

鉴于不同的细胞有不同的功能, Zhang课题组 ${ }^{[70]}$ 采用细胞膜融合的方法构建出红细胞膜-血小板复合 膜, 并将其用于纳米粒子的表面修饰. 研究结果表明, 
(a)
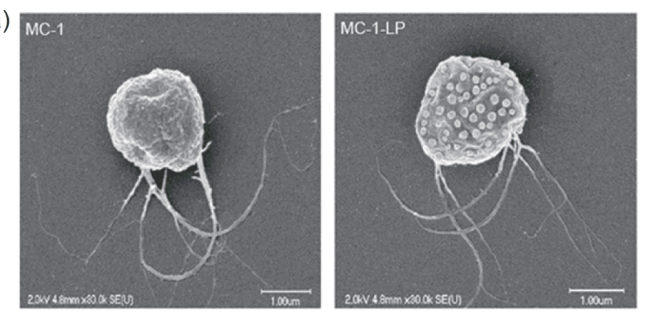

(c)

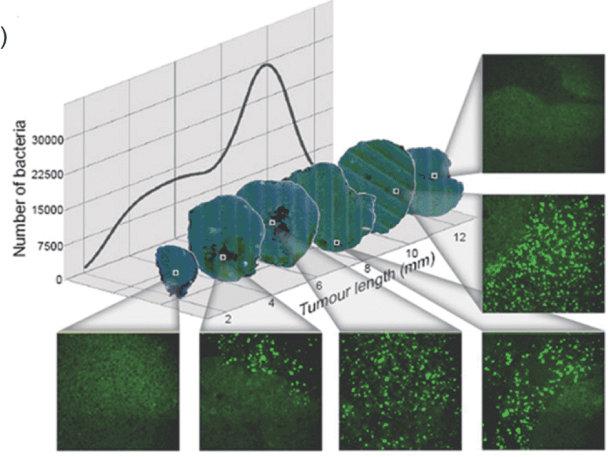

(b)

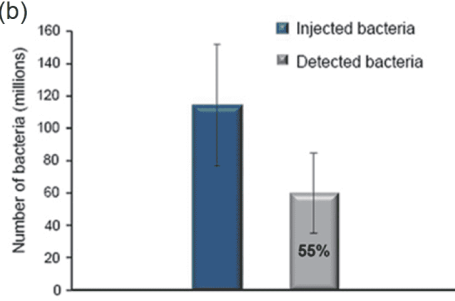

(d)

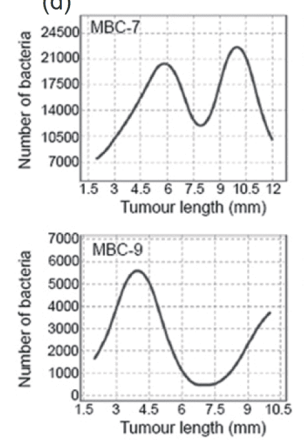

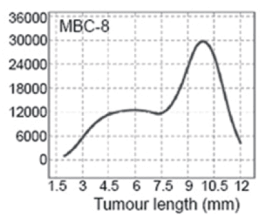

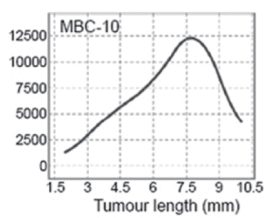

图 5 (a) MC-1 (左)及表面修饰了载药脂质体纳米粒子的MC-1 (右)细菌的扫描电子显微镜图; (b) 通过磁场作用在肿瘤部位 靶向富集的细菌数量与注射的细菌总量的统计结果对比图; (c) 肿瘤不同横切面处细菌的分布情况及相应的细菌的免疫荧光 染色图片; (d) 在不同的肿瘤当中, 细菌的分布情况(由于肿瘤的大小和形状及乏氧区域的不同, 因此造成细菌分布的差异) ${ }^{[61]}$ (网络版彩图)

Figure 5 (a) Scanning electron microscopy images of MC-1 and drug-containing liposome-loaded MC-1; (b) comparision of the bacteria numbers accumulated in the tumor site and their total injected numbers; (c) distribution of the bacteria in different tumor sections and the corresponding fluorescence images of each section. Bacteria were stained with green; (d) examples of bacteria distributions inside different tumors. The differences in the tumor size, shape and locations of the hypoxic regions among the various tumors lead to variations in the distributions of bacteria [61] (color online).

该方法所得到的复合膜包覆的纳米粒子不仅保留了红 细胞膜的体内长循环和血小板的肿瘤靶向的特性, 并 且具有良好的生物相容性, 为多功能性细胞仿生药物 递送平台的构建提供了新的技术支持.

\section{7 总结与展望}

基于生物细胞的仿生药物递送体系的出现为癌症 的治疗带来了极大的便利, 它有机结合了人工合成纳 米粒子和天然生物体系两者的优势. 一方面, 细胞/细 胞膜的包覆使纳米载药粒子具有更好的生物相容性、 长的血液循环时间及肿瘤靶向的特性; 另一方面, 纳米 载体的使用不仅可以提高抗肿瘤药物的利用效率, 降 低其副作用, 而且纳米粒子的材料及功能多样性也为 设计特定的纳米药物实现肿瘤的个性化治疗提供了更 多的选择. 本文简要介绍了近年来在基于红细胞、血 小板、免疫细胞、干细胞、细菌及细胞外泌体的生物
细胞仿生药物递送体系的设计构建及其在抗癌药物的 递送方面所做的研究工作(表1). 尽管取得了一些振奋 人心的成绩, 但是这些研究工作大都还处于临床前期 的探索阶段, 要将细胞仿生药物递送系统用于临床癌 症治疗, 仍然有许多问题需要解决. 首先, 细胞及细胞 膜的提取和纯化过程相对于人工合成材料更复杂、技 术要求更高, 如何确保在纳米药物的制备过程中细胞 膜结构的稳定及细胞生理机能的维持, 同时避免细菌 感染等污染的发生是细胞仿生纳米药物在大规模批量 生产中首要考虑的问题. 其次, 细胞及细胞膜包覆的纳 米药物在血液循环过程中是否会发生细胞膜脱落、药 物的泄露, 进而影响其功能的发挥是构建仿生药物需 要考虑的另一个重要问题. 当前, 关于细胞仿生的载 药系统在生物活体内稳定性的问题, 尚缺乏系统的研 究. 第三, 特定的膜结构如癌细胞膜、细菌表面的膜 蛋白和抗原物质是否会对机体带来不必要的损伤或诱 导癌基因的表达, 即仿生递药体系的生物安全性是其 
表 1 生物细胞仿生药物递送系统的制备方式、在癌症治疗领域中的应用及细胞特性汇总

Table 1 Summary of the sources and the unique properties of membrane, fabrication method, and their applications in cancer therapy of the cell membrane-mimetic nano-drugs

\begin{tabular}{|c|c|c|c|c|}
\hline 细胞 & 中心纳米粒子/药物 & 制备方式 & $\begin{array}{c}\text { 㿋症治疗 } \\
\text { 方面的应 } \\
\text { 用 }\end{array}$ & 主要特性 \\
\hline \multirow{4}{*}{ 红细胞 } & 载药介孔普鲁士蓝 ${ }^{[23]}$ & 表面包覆 & $\begin{array}{l}\text { 化疗/光热 } \\
\text { 治疗 }\end{array}$ & \multirow{4}{*}{$\begin{array}{c}\text { 体内长循环, } \\
\text { 提取方便, 易 } \\
\text { 于表面修饰 }\end{array}$} \\
\hline & ICG、阿霉素[24] & 低渗透析 & $\begin{array}{l}\text { 化疗/光热 } \\
\text { 治疗 }\end{array}$ & \\
\hline & 载药聚合物纳米粒子[25] & 表面包覆 & 化疗 & \\
\hline & 载药聚合物纳米粒子[28] & 表面包覆 & 免疫治疗 & \\
\hline 血小板 & 载药纳米凝胶 ${ }^{[33]}$ & 表面包覆 & 联合化疗 & $\begin{array}{l}\text { 血管损伤靶向 } \\
\text { 性, 靶向术后 } \\
\text { 残存癌细胞 }\end{array}$ \\
\hline \multirow{3}{*}{ 巨噬细胞 } & 环磷酰胺[34] & 跨膜运输 & 免疫治疗 & \multirow{3}{*}{$\begin{array}{c}\text { 肿瘤靶向性强, } \\
\text { 可深入至镏 } \\
\text { 之氧部位 }\end{array}$} \\
\hline & 金纳米棒[39] & 细胞内吞 & 光热治疗 & \\
\hline & 载药聚合物纳米粒子 [40] & 细胞内吞 & 化疗 & \\
\hline \multirow{2}{*}{ 中性粒细胞 } & 载药聚合物纳米粒子[45] & 表面包覆 & 化疗 & \multirow{2}{*}{$\begin{array}{c}\text { 炎症（肿瘤） } \\
\text { 靶向性, 穿过 } \\
\text { 血脑拼障 }\end{array}$} \\
\hline & 载药脂质体 ${ }^{[46]}$ & 细胞内吞 & 化疗 & \\
\hline \multirow{3}{*}{ 癌细胞 } & 载药聚合物纳米粒子[49] & 表面包覆 & 化疗 & \multirow{3}{*}{$\begin{array}{l}\text { 免疫逃逸, 同 } \\
\text { 源靶向性 }\end{array}$} \\
\hline & 载药聚合物纳米粒子[50] & 表面包覆 & 化疗 & \\
\hline & 载药金属-有机框架[51] & 表面包覆 & $\begin{array}{l}\text { 光动力/饥 } \\
\text { 饿疗法 }\end{array}$ & \\
\hline \multirow{2}{*}{ 干细胞 } & $\begin{array}{c}\text { 负载siRNA氧化铁纳米粒 } \\
\text { 子[58] }\end{array}$ & 表面包覆 & $\begin{array}{l}\text { 光热/基因 } \\
\text { 治疗 }\end{array}$ & \multirow{2}{*}{$\begin{array}{c}\text { 免疫原性低, } \\
\text { 肿瘤靶向, 穿 } \\
\text { 过血脑屏障 }\end{array}$} \\
\hline & $\begin{array}{c}\text { 载药聚合物纳米粒子/脂 } \\
\text { 质体 }{ }^{[59]}\end{array}$ & 细胞内吞 & - & \\
\hline 细菌 & 载药纳米脂质体 ${ }^{[66]}$ & $\begin{array}{l}\text { 表面共价 } \\
\text { 键合 }\end{array}$ & 化疗 & $\begin{array}{c}\text { 免疫原性低, } \\
\text { 肿瘤靶向 }\end{array}$ \\
\hline \multirow[b]{3}{*}{ 外泌体 } & 阿霉素[67] & 电穿孔 & 化疗 & \multirow{3}{*}{$\begin{array}{c}\text { 免疫原性低, } \\
\text { 尺寸小 (40- } \\
100 \mathrm{~nm}) \text {, 体 } \\
\text { 内长循环, 易 } \\
\text { 于与受体细胞 } \\
\text { 融合, 穿过血 } \\
\text { 脑屏障 }\end{array}$} \\
\hline & 紫杉醇[68] & $\begin{array}{l}\text { 超声共孵 } \\
\text { 育 }\end{array}$ & 化疗 & \\
\hline & $\operatorname{miRNA}{ }^{[69]}$ & 基因转染 & 基因治疗 & \\
\hline
\end{tabular}

在临床应用的关键也是当前面临的最重要的问题。相 信随着科学界对这些问题的研究和探讨的不断深入,
基于生物细胞的仿生药物递送体系必将会推动癌症的 精准医疗迈向新的阶段.

\section{参考文献}

1 Miller KD, Siegel RL, Lin CC, Mariotto AB, Kramer JL, Rowland JH, Stein KD, Alteri R, Jemal A. CA-A Cancer J Clin, 2016, 66: 271-289

2 Minchinton AI, Tannock IF. Nat Rev Cancer, 2006, 6: 583-592

3 Lammers T, Kiessling F, Hennink WE, Storm G. J Control Release, 2012, 161: 175-187

4 Ferrari M. Nat Rev Cancer, 2005, 5: 161-171

5 Peer D, Karp JM, Hong S, Farokhzad OC, Margalit R, Langer R. Nat Nanotech, 2007, 2: 751-760

6 Davis ME, Chen ZG, Shin DM. Nat Rev Drug Discov, 2008, 7: 771-782

7 Harris JM, Chess RB. Nat Rev Drug Discov, 2003, 2: 214-221

8 Knop K, Hoogenboom R, Fischer D, Schubert US. Angew Chem Int Ed, 2010, 49: 6288-6308

9 Turecek PL, Bossard MJ, Schoetens F, Ivens IA. J Pharm Sci, 2016, 105: 460-475

10 Lu J, Chuan X, Zhang H, Dai W, Wang X, Wang X, Zhang Q. Int J Pharm, 2014, 471: 525-535

11 Rao L, Xu JH, Cai B, Liu H, Li M, Jia Y, Xiao L, Guo SS, Liu W, Zhao XZ. Nanotechnology, 2016, 27: 085106 
12 Théry C, Ostrowski M, Segura E. Nat Rev Immunol, 2009, 9: 581-593

13 Tan S, Wu T, Zhang D, Zhang Z. Theranostics, 2015, 5: 863-881

14 Kroll AV, Fang RH, Zhang L. Bioconjugate Chem, 2017, 28: 23-32

15 Anselmo AC, Mitragotri S. J Control Release, 2014, 190: 531-541

16 Balasubramanian V, Correia A, Zhang H, Fontana F, Mäkilä E, Salonen J, Hirvonen J, Santos HA. Adv Mater, 2017, 29: 1605375

17 Fang RH, Jiang Y, Fang JC, Zhang L. Biomaterials, 2017, 128: 69-83

18 Aryal S, Hu CMJ, Fang RH, Dehaini D, Carpenter C, Zhang DE, Zhang L. Nanomedicine, 2013, 8: 1271-1280

19 Gao W, Hu CMJ, Fang RH, Luk BT, Su J, Zhang L. Adv Mater, 2013, 25: 3549-3553

20 Hu CMJ, Fang RH, Zhang L. Adv Healthc Mater, 2012, 1: 537-547

Piao JG, Wang L, Gao F, You YZ, Xiong Y, Yang L. ACS Nano, 2014, 8: 10414-10425

Chen W, Zeng K, Liu H, Ouyang J, Wang L, Liu Y, Wang H, Deng L, Liu YN. Adv Funct Mater, 2017, 27: 1605795

Sun X, Wang C, Gao M, Hu A, Liu Z. Adv Funct Mater, 2015, 25: 2386-2394

Zou Y, Liu Y, Yang Z, Zhang D, Lu Y, Zheng M, Xue X, Geng J, Chung R, Shi B. Adv Mater, 2018, 30: 1803717

Sahin U, Türeci Ö. Science, 2018, 359: 1355-1360

Farkona S, Diamandis EP, Blasutig IM. BMC Med, 2016, 14: 73

Guo Y, Wang D, Song Q, Wu T, Zhuang X, Bao Y, Kong M, Qi Y, Tan S, Zhang Z. ACS Nano, 2015, 9: 6918-6933

Gao M, Liang C, Song X, Chen Q, Jin Q, Wang C, Liu Z. Adv Mater, 2017, 29: 1701429

Buergy D, Wenz F, Groden C, Brockmann MA. Int J Cancer, 2012, 130: 2747-2760

Ortiz-Otero N, Mohamed Z, King M R. Platelet-based drug delivery for cancer applications. In: Dong C, Zahir N, Konstantopoulos K, Eds. Biomechanics in Oncology. Advances in Experimental Medicine and Biology, Vol. 1092. Cham: Springer, 2018. 235-251

Bambace NM, Holmes CE. J Thromb Haemost, 2011, 9: 237-249

Hu Q, Sun W, Qian C, Wang C, Bomba HN, Gu Z. Adv Mater, 2015, 27: 7043-7050

Zhang X, Wang J, Chen Z, Hu Q, Wang C, Yan J, Dotti G, Huang P, Gu Z. Nano Lett, 2018, 18: 5716-5725

Noy R, Pollard JW. Immunity, 2014, 41: 49-61

Joyce JA, Pollard JW. Nat Rev Cancer, 2009, 9: 239-252

Qian BZ, Pollard JW. Cell, 2010, 141: 39-51

Wynn TA, Chawla A, Pollard JW. Nature, 2013, 496: 445-455

An L, Wang Y, Lin J, Tian Q, Xie Y, Hu J, Yang S. ACS Appl Mater Interfaces, 2019, 11: 15251-15261

He X, Cao H, Wang H, Tan T, Yu H, Zhang P, Yin Q, Zhang Z, Li Y. Nano Lett, 2017, 17: 5546-5554

Kolaczkowska E, Kubes P. Nat Rev Immunol, 2013, 13: 159-175

Jenne CN, Wong CHY, Zemp FJ, McDonald B, Rahman MM, Forsyth PA, McFadden G, Kubes P. Cell Host Microbe, 2013, 13: 169-180

Coffelt SB, Wellenstein MD, de Visser KE. Nat Rev Cancer, 2016, 16: 431-446

Powell DR, Huttenlocher A. Trends Immunol, 2016, 37: 41-52

Kang T, Zhu Q, Wei D, Feng J, Yao J, Jiang T, Song Q, Wei X, Chen H, Gao X, Chen J. ACS Nano, 2017, 11: 1397-1411

Xue J, Zhao Z, Zhang L, Xue L, Shen S, Wen Y, Wei Z, Wang L, Kong L, Sun H, Ping Q, Mo R, Zhang C. Nat Nanotech, 2017, 12: 692-700

Fang RH, Hu CMJ, Luk BT, Gao W, Copp JA, Tai Y, O’Connor DE, Zhang L. Nano Lett, 2014, 14: 2181-2188

Hanahan D, Weinberg RA. Cell, 2011, 144: 646-674

Sun H, Su J, Meng Q, Yin Q, Chen L, Gu W, Zhang P, Zhang Z, Yu H, Wang S, Li Y. Adv Mater, 2016, 28: 9581-9588

Yang R, Xu J, Xu L, Sun X, Chen Q, Zhao Y, Peng R, Liu Z. ACS Nano, 2018, 12: 5121-5129

Li SY, Cheng H, Xie BR, Qiu WX, Zeng JY, Li CX, Wan SS, Zhang L, Liu WL, Zhang XZ. ACS Nano, 2017, 11: 7006-7018

Corsten MF, Shah K. Lancet Oncol, 2008, 9: 376-384

Stuckey DW, Shah K. Nat Rev Cancer, 2014, 14: 683-691

Mu X, Li J, Yan S, Zhang H, Zhang W, Zhang F, Jiang J. ACS Biomater Sci Eng, 2018, 4: 3895-3905

Roger M, Clavreul A, Venier-Julienne MC, Passirani C, Montero-Menei C, Menei P. Biomaterials, 2011, 32: 2106-2116

Martinez-Quintanilla J, Bhere D, Heidari P, He D, Mahmood U, Shah K. Stem Cells, 2013, 31: 1706-1714

Roger M, Clavreul A, Venier-Julienne MC, Passirani C, Sindji L, Schiller P, Montero-Menei C, Menei P. Biomaterials, 2010, 31: 8393-8401 

1779

\title{
Cell-derived biomimetic drug delivery systems for cancer therapy
}

\author{
Wenya He, Yan Zou ${ }^{*}$, Meng Zheng, Bingyang Shi ${ }^{*}$ \\ International Joint Centre for Biomedical Innovation, School of Life Sciences, Henan University, Kaifeng 475004, China \\ *Corresponding authors (email: yzou@henu.edu.cn; bs@henu.edu.cn)
}

\begin{abstract}
Nano-drugs hold great potential in the area of precision medicine. However, the easy recognition and fast clearance of nanoparticles by the immune systems has greatly hindered their application in cancer therapy. Inspired by the natural systems, cell-derived biomimetic drug delivery systems have aroused significant attention in recent years. This strategy, in which nano-drug carriers were surface-coated with endogenous cell membranes or co-incubated with live cells to form drug-carrying cells, organically combines the unique functionalities of cells and synthetic nanomaterials for effective delivery of therapeutic agents. On one hand, it can decrease the immunogenicity of nanodrugs, and prolong their in vivo blood circulation time. On the other hand, it will endow the nano-drugs with enhanced tumor targeting ability. In this review, we introduce the research progress of the application of cell-derived biomimetic drug delivery systems in cancer therapy, and discuss the challenges for additional breakthroughs in this field.
\end{abstract}

Keywords: biomimetic functionalization, nano-drug, cell, cancer therapy

doi: $10.1360 /$ SSC-2019-0068 